\title{
Evolution from Commensurability to Size-Effects Structures in Three-Dimensional Billiards
}

\author{
N. M. Sotomayor, G. M. Gusev, and J. R. Leite. \\ Instituto de Física da Universidade de São Paulo, CP 66318, CEP 05315-970, SP, Brazil
}

Received on 31 March, 2003

\begin{abstract}
We calculated the classical electron dynamics of three-dimensional electrons in a billiard system in tilted magnetic field and analyzed the evolution of trajectories in phase space by means of Poincaré space of sections. The low field magnetoresistance $\rho_{x x}$ was calculated through linear response theory and found that nonlinear resonances between the cyclotron radius $R_{c}$, the antidot lattice period $a$, and the well width $W$, are reflected in the observed magnetoresistance peaks.
\end{abstract}

\section{Introduction}

Advances in epitaxial growth and microfabrication technology have led to the experimental realization of a quasi-three-dimensional electron billiard system, in wide $A l_{x} G a_{1-x} A s / G a A s$ parabolic quantum wells (PQW), with high mobility [1]. These systems contain a rectangular array of cylindrical voids, with sub-micron diameter, patterned across the PQW and barrier layers. Due to the combined influence of the electron magneto-focusing effect and boundary scattering the electron motion leads to different kinds of anomalous peaks in the experimental $\rho_{x x}$, at low field, performed in tilted magnetic field [1]. These measurements also demonstrated that the oscillations do not shift continuously toward higher values of the field as in the case of antidots lattices in strictly two-dimensional systems, instead they suffer a sudden transformation into structures that were attributed to galvano-magnetic size effects. However, for the proper understanding of the experimental data, and for a determination of the role of the electron trajectories, it is necessary to calculate the carrier dynamics of a thin film with a lattice of periodical voids confined, axially, by a parabolic potential.

\section{Tilted field dynamics}

For the present work, we use a classical approximation for the dynamics of electrons in a 3D billiard under the influence of tilted magnetic field. Indeed the full quantum-mechanical calculations of the energy spectrum and conductivities are necessary to describe the experimental results. However, we believe that classical calculations can reproduce the $\rho_{x x}$ curves including peak positions of the commensurate resonances. In this work we compare only positions of the peaks of magnetoresistance with experimental traces and consider different 3D regular trajectories and its contribution to the conductivity. For this purpose classical method works quite effectively. In order to model the electron dynamics in an array of cylindrical voids, we depart from the single-particle Hamiltonian for a three-dimensional electron,

$$
H=\frac{1}{2 m^{*}}(\vec{p}-e \vec{A})^{2}+U_{A D}(x, y)+U_{w}(z)
$$

we chose an angle dependent potential vector given by $\vec{A}=(1 / 2) B[z \cos \theta-y \sin \theta, x \sin \theta,-x \cos \theta]$, where $e$ is the electron charge, $m^{*}$ is the electron effective mass, and $\Theta$ is the angle between the magnetic field vector and the surface of the PQW sample. The modulation of the electrostatic potential has no dependence of the $z$ coordinate and can be simulated by the expression, $U_{A D}(x, y)=$ $U_{0}\{\cos (2 \pi x / a) \cos (2 \pi y / a)\}^{\beta}$, where $\beta$ is an even integer which stands for the steepness of the potential, $U_{0}$ is the maximum amplitude, and $a$ is the antidot lattice period. For our calculations we used the soft-potential picture and take $\beta=6-8 . U_{0}$ is assumed to be 1.6 times the Fermi energy. The confinement along the $z$ direction due to the potential of the well and barriers is introduced by means of the expression: $U_{w}(z)=\left(1 / 2 m^{*}\right) \Omega^{\gamma} z^{\gamma}$, where $\Omega$ and $\gamma$ are parameters that may be used to fit different profiles of the confining potential. We used dimensionless variables defined by: $\tilde{x}=x / a, \tilde{y}=y / a, \tilde{z}=z / a, \tilde{t}=t / \tau_{0}, \tilde{H}=H / E_{F}$, and $\tilde{U}=U / E_{F}$, where $E_{F}$ is the three dimensional Fermi energy. The time is scaled by: $\tilde{\tau}_{0}=\left(m^{*} a^{2} / 2 E_{F}\right)^{1 / 2}$, where $\tau_{0}$ is the time that an electron delays when travel a distance equivalent to $a$ running at the Fermi velocity. The magnetic field is scaled by: $B_{0}=(2 / e a)\left(2 m^{*} E_{F}\right)^{1 / 2}$, where $B_{0}$ is the value of the magnetic field which corresponds to a cyclotron radius equivalent to half the lattice period ( $R_{c}=a / 2$ ). By performing these substitutions, and omitting tildes, the dimensionless Hamiltonian reads: 


$$
\begin{array}{r}
\left(\frac{d x}{d t}+\frac{B}{B_{0}}(z \cos (\theta)-y \sin (\theta))^{2}+\right. \\
\left(\frac{d y}{d t}-\frac{B}{B_{0}} x \sin (\theta)\right)^{2}+\left(\frac{d z}{d t}+\frac{B}{B_{0}} x \cos (\theta)\right)^{2}+ \\
U_{A D}+U_{w} .
\end{array}
$$

The equations of motion for the system are given by:

$$
\begin{array}{r}
v_{x}=2\left(\frac{d x}{d t}-\frac{B}{B_{0}}(z \cos (\theta)-y \sin (\theta))\right. \\
v_{y}=2\left(\frac{d y}{d t}-\frac{B}{B_{0}} x \sin (\theta)\right) \\
v_{z}=2\left(\frac{d z}{d t}+\frac{B}{B_{0}} x \cos (\theta)\right) \\
\dot{v}_{x}=\frac{1}{2} \frac{B}{B_{0}}\left(v_{x} \sin (\theta)-v_{z} \cos (\theta)\right)-\frac{\partial U_{A D}}{\partial x} \\
\dot{v}_{y}=-\frac{B}{B_{0}} \sin (\theta) v_{x}-\frac{\partial U_{A D}}{\partial y} \\
\dot{v}_{z}=\frac{B}{B_{0}} \cos (\theta) v_{x}-\frac{\partial U_{w}}{\partial z}
\end{array}
$$

A sixth-order Runge-Kutta-Verner method was employed to integrate numerically these equations of motion. When $\theta=90^{\circ}$, the Hamilton function separates and the motion in the $x-y$ plane and in $z$ direction can be treated separately. There are two additional integrals of motion, the energy of the $z$-motion and the $x y$-motion, that are conserved individually. The resulting three-dimensional motion is a combination of a two-dimensional chaotic motion with the completely integrable motion along $z$ direction. Furthermore, the uncoupling of the motion also leads to chaotic motion in the $x-y$ plane for different energies $E_{z}$, where $E_{F}=E_{x-y}-E_{z}, E_{F}$ is the Fermi energy, $E_{x-y}$ is the in-plane energy and $E_{z}$ the energy along $z$ direction. Thus the dynamics is mixed and, there is a coexistence of regular and chaotic trajectories. When the magnetic field is tilted $0^{\circ}<\theta<90^{\circ}$, a coupling of the degrees of freedom appears and the system undergoes a transition to chaos. The system has three degrees of freedom and six dynamical variables $\left(x, y, z, v_{x}, v_{y}, v_{z}\right)$, the electron trajectories in phase space are confined to five-dimensional surface of constant energy, in this space, a Poincaré section can be defined as the intersection of the orbits with a subspace with dimension $2 n-2=4$, in correspondence with the mapping in two-dimensional systems. For a 3D integrable Hamiltonian system, with a $6 \mathrm{D}$ phase space, the integrable surfaces are tree-dimensional and the points belonging to the fourthdimensional mapping must lie on 2D invariant tori. If the system turns non integrable, and the Kolmogorov-ArnoldMoser (KAM) theorem [2] is still applicable, again the integrable surfaces are 3D and the two-dimensional tori remain on the 4D space.
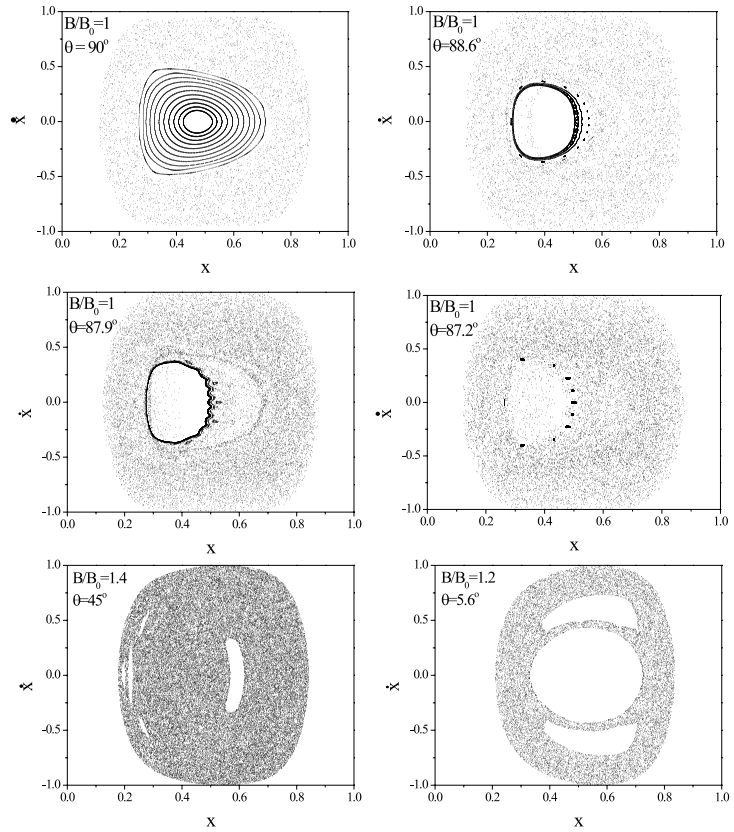

Figure 1. 2D Poincaré projections of 4D space of sections for increasing tilted fields and different magnetic field values.

Figure 1, show six 2D projections $(x, \dot{x})$ of the 4D space of section $(x, y, \dot{x}, \dot{y})$ for a set of trajectories originated by a particular ensemble of initial conditions, for the same value of the normalized magnetic field $B / B_{0}$ and for different values of the angle $\theta$. This mapping was calculated for a ratio $d / a=0.22$ ( $d$ is the antidot diameter), that corresponds to an experimental sample with lattice periodicity $a=0.5 \mu \mathrm{m}$. For the calculation, the initial position of the $x, z$ coordinates, and all velocities were maintained constant. After we vary the initial position of the $y$ coordinate in steps of $0.01 a$ along one of the sides of the unit cell defined by the antidot lattice period $a$, The mappings correspond to the two-dimensional case of Poincaré surfaces of the section at $y=y_{0}$ which is the intersection of the energy surface with the plane $y=y_{0}$ denoted by $[y(\bmod 1)=0]$. When $\theta=90^{\circ}$ the islands correspond to regular orbits revolving around a cylindrical void and after multiple specular reflections, with the well interfaces, the electrons remain pinned by the antidot. The group of small islands, located between the innermost and outermost islands, correspond to a single quasi-periodic trajectories, and indicates that almost all phase space is filled by integrable KAM curves. As the tilted field increases the KAM curves are gradually deformed and destroyed and the chaotic component appears. Therefore, the distribution of chaotic points, referred as "consequents" in literature [3], fills the phase portrait at $B / B_{0}=1$. However, for larger values of the tilted field we still observe remnants of KAM curves and stable islands for higher values of $B / B_{0}$. This feature demonstrates that nonlinear resonances are still responsible for the shifting of the commensurability peaks. Another important feature is that for tilted angles less than $\theta \approx 22.5^{\circ}$ we found again surviving KAM curves and stable islands for magnetic field values close around $B / B_{0}=1$, this is due to 
the fact that the degree of coupling decrease and the system becomes again integrable in the case when the magnetic field is parallel to the $y$ direction. In PQW in tilted field, a new third length scale given by the well width $W$ plays an important role by allowing extra geometrical resonances, which may be responsible for the anomalous peaks in parallel magnetic field, as demonstrated by the $\rho_{x x}$ calculations showing in the next paragraph. In order to calculate the magnetoresistance we used classical linear response theory where the Ohmic conductivity $\sigma_{i j}$ is given, by the expression [2]: $\sigma_{i j}=\frac{m^{*} e^{2}}{\pi \hbar^{2}} \int_{0}^{\infty}\left\langle v_{i}(t) v_{j}(t=0)\right\rangle_{\Gamma} e^{-\frac{t}{\tau}} d t$, where $\hbar$ is the reduced Planck constant and $\left\langle v_{i}(t) v_{j}(0)>_{\Gamma}\right.$ is the velocity-velocity correlation function double averaged over phase space $\Gamma$, the indices $i$ and $j$ stand for the $x$ and $y$ direction, respectively. The presence of impurity scattering is included through the electron mean scattering time $\tau$, where the probability of an electron not suffering a collision within the time interval $[0, \mathrm{t}]$ is given by $e^{-t / \tau}$. From the numerical computation of the conductivity tensors $\sigma_{x x}$ and $\sigma_{x y}$, we are able to determine the longitudinal $\rho_{x x}$ and transverse $\rho_{x y}$ resistivities in tilted magnetic field. Figure 2(a) shows the calculated magnetoresistance for a ratio $d / a=0.22$ and for a well with $W=a$, for different values of the angle $\theta$. In perpendicular field we obtain two peaks corresponding to the conditions $R_{c}=a / 2$ and $R_{c} \approx 2.0 a$ in correspondence which is experimentally observed. As the angle $\theta$ decreases, the peaks shift toward higher values of the normalized field. If the well is narrow $W=0.4 a$ the behavior of the main peak tends to the $\sin ^{-1}(\theta)$ law of two-dimensional antidots in tilted magnetic field (see fig. 2(b)). However, when the well width is increased $W=a$ we obtain a sudden turn at the values of $\theta \approx 22.5^{\circ}$, that agrees with the observed in experiments (see fig. 2(c)). These results indicate that the coexistence of geometrical resonances between the antidot period and the well width produce the anomalous behavior of the commensurability peaks in tilted field. We would like to thank FAPESP, Brazilian Funding Agency by financial support and to the LCCA-USP by computational facilities.

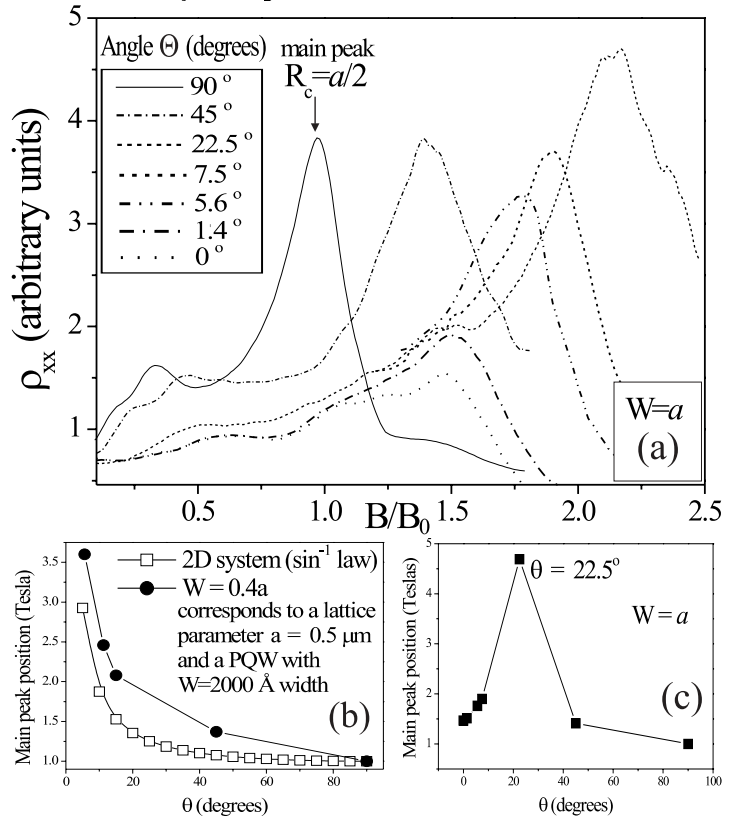

Figure 2. (a) Magnetoresistance in tilted field for an antidot sample of periodicity $a$ and $W=a$, (b) evolution of the main comensurability peak position in tilted field for $W=0.4 a$, (c) the same evolution that in (b) for a sample with $W=a$.

\section{References}

[1] N. M. Sotomayor, G. M. Gusev, J. R. Leite, A. A. Bykov, L. V. Litvin, N. T. Moshegov, A. I. Toropov, O. Estibals, and J. C. Portal, Physical Review B, in press (2003).

[2] R. Fleischmann, T. Geisel, and R. Ketzmerick, Phys. Rev. Lett. 68, 1367 (1992).

[3] L. Martinet and P. Magnenat, Astron. \& Astrophys. 96, 68 (1981). 